\title{
Liver Enzymes and Risk of Stroke: The Atherosclerosis Risk in Communities (ARIC) Study
}

\author{
Angela Ruban, ${ }^{\mathrm{a}}$ Natalie Daya, ${ }^{\mathrm{b}}$ Andrea L.C. Schneider, ${ }^{\mathrm{c}}$ Rebecca Gottesman, ${ }^{\mathrm{b}, \mathrm{c}}$ Elizabeth Selvin, ${ }^{\mathrm{b}}$ \\ Josef Coresh, ${ }^{\mathrm{b}}$ Mariana Lazo, ${ }^{\mathrm{b}}$ Silvia Koton ${ }^{\mathrm{a}, \mathrm{b}}$ \\ ${ }^{a}$ Department of Nursing, Sackler Faculty of Medicine, Tel Aviv University, Tel Aviv, Israel \\ ${ }^{b}$ Department of Epidemiology, Johns Hopkins University School of Medicine, Baltimore, MD, USA \\ 'Department of Neurology, Johns Hopkins University School of Medicine, Baltimore, MD, USA
}

Background and Purpose Liver enzymes (aspartate aminotransferase [AST], alanine aminotransferase [ALT], and gamma-glutamyl transpeptidase [GGT]) are glutamate-regulatory enzymes, and higher glutamate levels correlated with worse prognosis of patients with neurotrauma. However, less is known about the association between liver enzymes and incidence of stroke. We evaluated the association between serum levels of AST, ALT, and GGT and incidence of stroke in the Atherosclerosis Risk in Communities (ARIC) study cohort from 1990 to 1992 through December 31, 2016. Methods We included 12,588 ARIC participants without prevalent stroke and with data on liver enzymes ALT, AST, and GGT at baseline. We used multivariable Cox regression models to examine the associations between liver enzymes levels at baseline and stroke risk (overall, ischemic stroke, and intracerebral hemorrhage [ICH]) through December 31, 2016, adjusting for potential confounders.

Results During a median follow-up time of 24.2 years, we observed 1,012 incident strokes (922 ischemic strokes and $90 \mathrm{ICH}$ ). In age, sex, and race-center adjusted models, the hazard ratios (HRs; 95\% confidence intervals [Cls]) for the highest compared to lowest GGT quartile were 1.94 (95\% $\mathrm{Cl}, 1.64$ to 2.30) for all incident stroke and 2.01 (95\% Cl, 1.68 to 2.41$)$ for ischemic stroke, with the results supporting a dose-response association ( $P$ for linear trend $<0.001$ ). Levels of AST

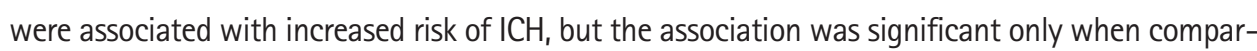
ing the third quartile with the lowest quartile (adjusted $\mathrm{HR}, 1.82 ; 95 \% \mathrm{Cl}, 1.06$ to 3.13 ).

Conclusions Elevated levels of GGT (within normal levels), independent of liver disease, are associated with higher risk of incident stroke overall and ischemic stroke, but not ICH.

Keywords Stroke; Incidence; Liver enzymes; Atherosclerosis Risk in Communities; Gamma-glutamyltransferase; Aspartate aminotransferases; Alanine transaminase
Correspondence: Angela Ruban

Department of Nursing, Steyer School of Health Professions, Sackler Faculty of Medicine, and Sagol School of Neuroscience, Tel Aviv University, Tel Aviv 69978, Israel

Tel: +972-3-6406259

Fax +972-3-6409496

E-mail: angellruban@tauex.tau.ac.il https://orcid.org/0000-0003-10918854

Received: January 26, 2020

Revised: May 26, 2020

Accepted: August 13, 2020

\section{Introduction}

Approximately 800,000 Americans have a stroke each year, and the overall stroke prevalence in the United States population is about 2.5\%. ${ }^{1}$ Although adjusted mortality rates for both isch- emic and hemorrhagic stroke globally declined between 1990 and 2015, the total number of people with stroke increased each year. ${ }^{1}$ Therefore, continuing research on the potential risk factors is very important for preventing stroke.

Previous studies have suggested that dysregulation of the 
glutamate metabolism is a primary contributor to neuronal damage in neurotrauma. ${ }^{2,3}$ Furthermore, few studies have demonstrated associations between blood glutamate and glutamate-regulatory enzymes levels, and risk of stroke. ${ }^{4-6}$ In a prospective case-cohort study, higher blood glutamate levels were associated with increased risk of nonfatal strokes in a population with either type 2 diabetes mellitus or three major risk factors for cardiovascular diseases. ${ }^{4}$

Aspartate aminotransferase (AST), also known as glutamateoxaloacetate transaminase (GOT1), alanine aminotransferase (ALT), also known as glutamate-pyruvate transaminase (GPT), and gamma-glutamyl transpeptidase (GGT) are commonly known for their role for identifying hepatic injuries. ${ }^{7.8}$ Less known are the physiologic roles of AST and ALT as regulators of glutamate metabolism, by which glutamate is converted to alpha-ketoglutarate and L-aspartate or L-alanine. As a result, higher levels of AST and ALT results in decreased glutamate levels in blood and vice versa. ${ }^{9-11}$

The physiological function of GGT is to regulate the antioxidant homeostasis through recycling extracellular glutathione (GSH). ${ }^{12,13}$ It plays an important role in the metabolism of GSH and glutamate. ${ }^{13,14}$ GGT has emerged as a risk factor for allcause and cardiovascular mortality in population-based studies, independent of liver disease and alcohol intake. ${ }^{15,16} \mathrm{GSH}$, a well-known antioxidant agent, has been suggested as a physiological reservoir for glutamate. ${ }^{13,17}$ Therefore, in contrast to high levels of AST and ALT that result in lower levels of blood glutamate, increased GGT levels causes increased synthesis of glutamate. ${ }^{17,18}$

The limited number of studies assessing associations between levels of AST, ALT, GGT and risk of stroke have showed inconsistent results. ${ }^{6,79,20}$ In addition, while most studies have used ALT and AST as surrogates of non-alcoholic fatty liver disease (NAFLD), and GGT as a marker of excessive alcohol consumption and a potential marker for oxidative stress, ${ }^{14}$ they mention that potential mechanisms for this association have not been suggested. We hypothesized that levels of glutamateregulatory enzymes play a role in the occurrence of stroke, in part because of their role in glutamate metabolism. To test this hypothesis, we studied the association between blood levels of ALT, AST, and GGT and incidence of stroke in the Atherosclerosis Risk in Communities (ARIC) study cohort.

\section{Methods}

\section{Study population}

The ARIC study is a community-based cohort, including 15,792 individuals aged 45 to 64 at baseline (1987 to 1989). Partici- pants were recruited from four United States communities (Forsyth County, NC; Jackson, MS; Minneapolis, MN; and Washington County, MD). ${ }^{21}$ Liver enzymes were first measured at ARIC visit 2 (1990 to 1992). Among 14,348 ARIC visit 2 participants, 275 reported prevalent stroke and were excluded from this analysis. We also excluded participants with missing data on AST, ALT, or GGT ( $n=955)$, race other than white or black, blacks in Minneapolis, MN and Washington County, MD $(n=95)$, and those with missing data on the following covariates: body mass index (BMI), smoking status, alcohol use, education levels, systolic blood pressure, hypertension medication, coronary heart disease (CHD), diabetes, and total cholesterol $(n=405)$. Thus, 12,588 ARIC participants were included in the present analysis.

\section{Ascertainment of stroke}

An incident stroke was defined as the first stroke diagnosed from baseline (1990 to 1992) through December 31st, 2016 (median [interquartile range, IQR] follow-up of 24.2 [16.7 to 24.5] years). Stroke events were identified during ARIC visits, annual (semiannual since 2012) telephone calls or through surveillance of hospitals in the community. Stroke hospitalizations records with the following International Classification of Diseases, 9th revision (ICD-9) codes 430-438 (from 1990 to 1997) and ICD-9 codes $430-436$ or ICD-10 codes G45.X, I60.X, 161.X, I62.X, I63.X, I65.X, I66.X, I67.X (from 1998 until December 31,2016$)$ were reviewed in detail. Subarachnoid hemorrhage events were not included $(n=30)$. Stroke events were adjudicated by physician reviewers as definite or probable ischemic stroke or definite or probable intracerebral hemorrhage $(\mathrm{ICH}){ }^{22}$

\section{Measurement of liver enzymes}

ALT, AST, and GGT levels were measured in ARIC visit 2 (1990 to 1992) serum samples (stored at $-80^{\circ} \mathrm{C}$ since collection) using the Roche Modular P Chemistry analyzer and reagents from Roche Diagnostics (Indianapolis, IN). Using split samples collected at visit 2 and stored, the calculated coefficients of variation, were 16\% for ALT, 6\% for AST, and 4\% for GGT.

\section{Additional variables}

Sociodemographic information was collected at baseline. Weight and height were measured using standardized protocols and BMI was calculated as weight $(\mathrm{kg}) /$ height $\left(\mathrm{m}^{2}\right)$. Smoking status and alcohol use were self-reported, and categorized as never, former or current. Prevalent diabetes was defined as self-reported diagnosis by physician, diabetes medication use, fasting glucose $\geq 126 \mathrm{mg} / \mathrm{dL}$, or glycated hemoglobin $\geq 6.5 \%$. 
Prevalent hypertension was defined as average systolic blood pressure $\geq 140 \mathrm{~mm} \mathrm{Hg}$ or average diastolic blood pressure $\geq 90$ $\mathrm{mm} \mathrm{Hg}$ or use of hypertension medication. CHD was defined as self-reported physician diagnosis of myocardial infarction or coronary revascularization at visit 1 , or by adjudicated hospitalized CHD events occurring between visit 1 and 2 .

\section{Statistical analyses}

Baseline levels of ALT, AST, and GGT were categorized into sex- specific quartiles for each enzyme. We used descriptive statistics to characterize participants at baseline by quartiles of liver enzymes. Differences in baseline characteristics by quartiles of liver enzymes were tested with chi-square tests for categorical characteristics and analysis of variance (ANOVA) for continuous characteristics. The cumulative incidence of stroke by baseline quartiles of liver enzymes levels was assessed using Kaplan-Meier curves for total stroke, ischemic stroke and ICH. We used multivariable adjusted Cox proportional hazards mod-

Table 1. Characteristics of the study population by quartiles of GGT level at baseline, the ARIC study, visit 2, 1990-1992

\begin{tabular}{|c|c|c|c|c|c|c|}
\hline Characteristic & Total & GGT (01) & GGT (02) & GGT (03) & GGT (04) & $P$ \\
\hline Number & 12,588 & 3,618 (28.7) & $2,890(23.0)$ & $3,083(24.5)$ & 2,997 (23.8) & \\
\hline Age (yr) & $56.9 \pm 5.7$ & $56.4 \pm 5.7$ & $57.0 \pm 5.7$ & $57.2 \pm 5.8$ & $56.8 \pm 5.6$ & $<0.001$ \\
\hline Minneapolis Whites & $3,454(27.4)$ & $1,206(33.3)$ & $827(28.6)$ & $769(24.9)$ & $652(21.8)$ & $<0.001$ \\
\hline Jackson Blacks & $2,689(21.4)$ & $380(10.5)$ & 539 (18.7) & $784(25.4)$ & 986 (32.9) & \\
\hline Washington Co. Whites & $3,194(25.4)$ & $966(26.7)$ & 768 (26.6) & $747(24.2)$ & $713(23.8)$ & \\
\hline Forsyth Co. Blacks & $333(2.7)$ & $53(1.5)$ & $71(2.5)$ & $99(3.2)$ & 110 (3.7) & \\
\hline Forsyth Co. Whites & $2,918(23.2)$ & $1,013(28.0)$ & $685(23.7)$ & $684(22.2)$ & 539 (17.9) & \\
\hline Female sex & $7,084(56.3)$ & $2,152(59.5)$ & 1,531 (53.0) & $1,732(56.2)$ & $1,669(55.7)$ & $<0.001$ \\
\hline BMI $\left(\mathrm{kg} / \mathrm{m}^{2}\right)$ & $28.0 \pm 5.4$ & $25.9 \pm 4.3$ & $27.7 \pm 5.2$ & $29.1 \pm 5.6$ & $29.6 \pm 5.7$ & $<0.001$ \\
\hline $\mathrm{SBP}(\mathrm{mm} \mathrm{Hg})$ & $121.2 \pm 18.5$ & $116.2 \pm 17.7$ & $120.7 \pm 18.0$ & $123.3 \pm 17.9$ & $125.6 \pm 19.1$ & $<0.001$ \\
\hline $\mathrm{DBP}(\mathrm{mm} \mathrm{Hg})$ & $72.1 \pm 10.2$ & $69.9 \pm 9.9$ & $72.0 \pm 9.8$ & $72.9 \pm 10.0$ & $74.0 \pm 10.7$ & $<0.001$ \\
\hline Hypertension & 4,957 (39.4) & 973 (26.9) & $1,063(36.8)$ & $1,360(44.1)$ & $1,561(52.1)$ & $<0.001$ \\
\hline Antihypertensive medication use & $4,023(32.0)$ & $772(21.4)$ & $856(29.6)$ & $1,119(36.3)$ & $1,275(42.5)$ & $<0.001$ \\
\hline $\mathrm{HDL}-\mathrm{C}(\mathrm{mg} / \mathrm{dL})$ & $47.0(37.0-59.0)$ & $51.0(41.0-63.0)$ & $46.0(37.0-58.0)$ & $45.0(37.0-56.0)$ & $45.0(36.0-57.0)$ & $<0.001$ \\
\hline LDL-C (mg/dL) & $131.0(108.8-155.4)$ & $125.0(105.0-148.0)$ & $131.5(110.0-155.0)$ & $134.1(111.2-159.2)$ & $134.8(109.4-159.6)$ & $<0.001$ \\
\hline Triglycerides (mg/dL) & 114.0 (82.0-162.0) & $97.0(72.0-133.0)$ & $112.0(81.0-154.0)$ & $122.0(88.0-175.0)$ & 135.0 (95.0-201.0) & $<0.001$ \\
\hline Total cholesterol (mg/dL) & $207.0(183.0-233.0)$ & $200.0(179.0-224.0)$ & 205.0 (183.0-230.0) & $211.0(187.0-237.0)$ & $215.0(188.0-243.0)$ & $<0.001$ \\
\hline Lipid lowering medication use & 790 (6.3) & $214(5.9)$ & $172(6.0)$ & $186(6.0)$ & $218(7.3)$ & 0.082 \\
\hline Diabetes & $1,970(15.6)$ & $215(5.9)$ & $326(11.3)$ & $581(18.9)$ & $848(28.3)$ & $<0.001$ \\
\hline Prevalent CHD & $683(5.4)$ & $178(4.9)$ & $163(5.6)$ & $161(5.2)$ & $181(6.0)$ & 0.211 \\
\hline Smoking status & & & & & & 0.001 \\
\hline Current & $2,751(21.9)$ & 749 (20.7) & $632(21.9)$ & $671(21.8)$ & $699(23.3)$ & \\
\hline Former & $4,764(37.8)$ & $1,330(36.8)$ & $1,148(39.7)$ & $1,208(39.2)$ & $1,078(36.0)$ & \\
\hline Never & $5,073(40.3)$ & $1,539(42.5)$ & $1,110(38.4)$ & 1,204 (39.1) & $1,220(40.7)$ & \\
\hline Drinking status & & & & & & $<0.001$ \\
\hline Current & 7,169 (57.0) & 2,152 (59.5) & $1,649(57.1)$ & $1,681(54.5)$ & $1,687(56.3)$ & \\
\hline Former & 2,595 (20.6) & $689(19.0)$ & 627 (21.7) & $651(21.1)$ & $628(21.0)$ & \\
\hline Never & 2,824 (22.4) & 777 (21.5) & 614 (21.2) & $751(24.4)$ & $682(22.8)$ & \\
\hline AST (U/L) & $20.0(17.0-23.0)$ & $18.0(16.0-21.0)$ & $19.0(16.0-22.0)$ & $20.0(17.0-24.0)$ & $22.0(19.0-27.0)$ & $<0.001$ \\
\hline ALT (U /L) & $14.0(11.0-19.0)$ & $12.0(9.0-15.0)$ & $14.0(11.0-18.0)$ & $15.0(12.0-20.0)$ & $19.0(14.0-26.0)$ & $<0.001$ \\
\hline GGT (U/L) & $21.0(14.0-33.0)$ & $12.0(10.0-14.0)$ & $18.0(16.0-22.0)$ & $26.0(22.0-30.0)$ & $50.0(40.0-72.0)$ & $<0.001$ \\
\hline
\end{tabular}

Values are presented as number (\%), mean \pm standard deviation, or median (interquartile range). GGT range (U/L): men $01<19,02$ 19-25, 03 26-38, 04 $\geq 39$; women $01<14 ; 02$ 14-18; 03 19-29, $04 \geq 39$.

GGT, gamma-glutamyl transpeptidase; ARIC, Atherosclerosis Risk in Communities; BMI, body mass index; SBP, systolic blood pressure; DBP, diastolic blood pressure; HDL-C, high-density lipoprotein cholesterol; LDL-C, low density lipoprotein cholesterol; CHD, coronary heart disease; AST, aspartate aminotransferase; ALT, alanine aminotransferase. 
els to estimate adjusted hazard ratios (HRs) of stroke overall and by stroke type, using sex-specific quartiles of liver enzymes as the exposure. Tests of the proportionality assumptions showed no significant deviations for liver test quartiles. Linear trends across the quartiles of liver enzymes were tested by including the quartiles in the models as continuous variable. We constructed three models with progressive adjustment: Model 1 included age, sex, and race-center (whites, Washington County; whites, Minneapolis; blacks, Jackson; blacks, Forsyth County; and whites, Forsyth County) as covariates; Model 2 included all variables in Model 1 as well as BMl, alcohol-drinking, smoking, and education levels; Model 3 included all variables in Model 2 and lipids levels (total cholesterol and highdensity lipoprotein cholesterol), systolic blood pressure, hypertension medication use, lipid lowering medication use, prevalent $\mathrm{CHD}$, and prevalent diabetes. We also examined models 2 and 3 with use of hypertension medication, diabetes, CHD, cholesterol-lowering medications use, alcohol use, and smoking status modeled as time-varying covariates.

To characterize the shape of the associations of ALT, AST, and GGT levels with stroke risk, we used restricted cubic spline models (truncated at the 1st and 99th percentiles) with knots at the 5th, 35th, 65th, and 95th percentiles of each liver enzyme, and models were centered at 10th percentile of each enzyme. $^{23}$

We performed sensitivity analyses excluding individuals with presumed NAFLD at baseline $(n=530)$. Presumed NAFLD was defined as elevated level of AST ( $\geq 31 \mathrm{U} / \mathrm{L}$ for women or $\geq 37 \mathrm{U} / \mathrm{L}$ for men) or ALT ( $\geq 31 \mathrm{U} / \mathrm{L}$ for women or $\geq 41 \mathrm{U} / \mathrm{L}$ for men) in participants who reported never or moderate alcohol drinking $(\leq 21$ drinks/week for men, and $\leq 14$ drinks/week for women).

To evaluate the incidence of stroke with a competing risk of
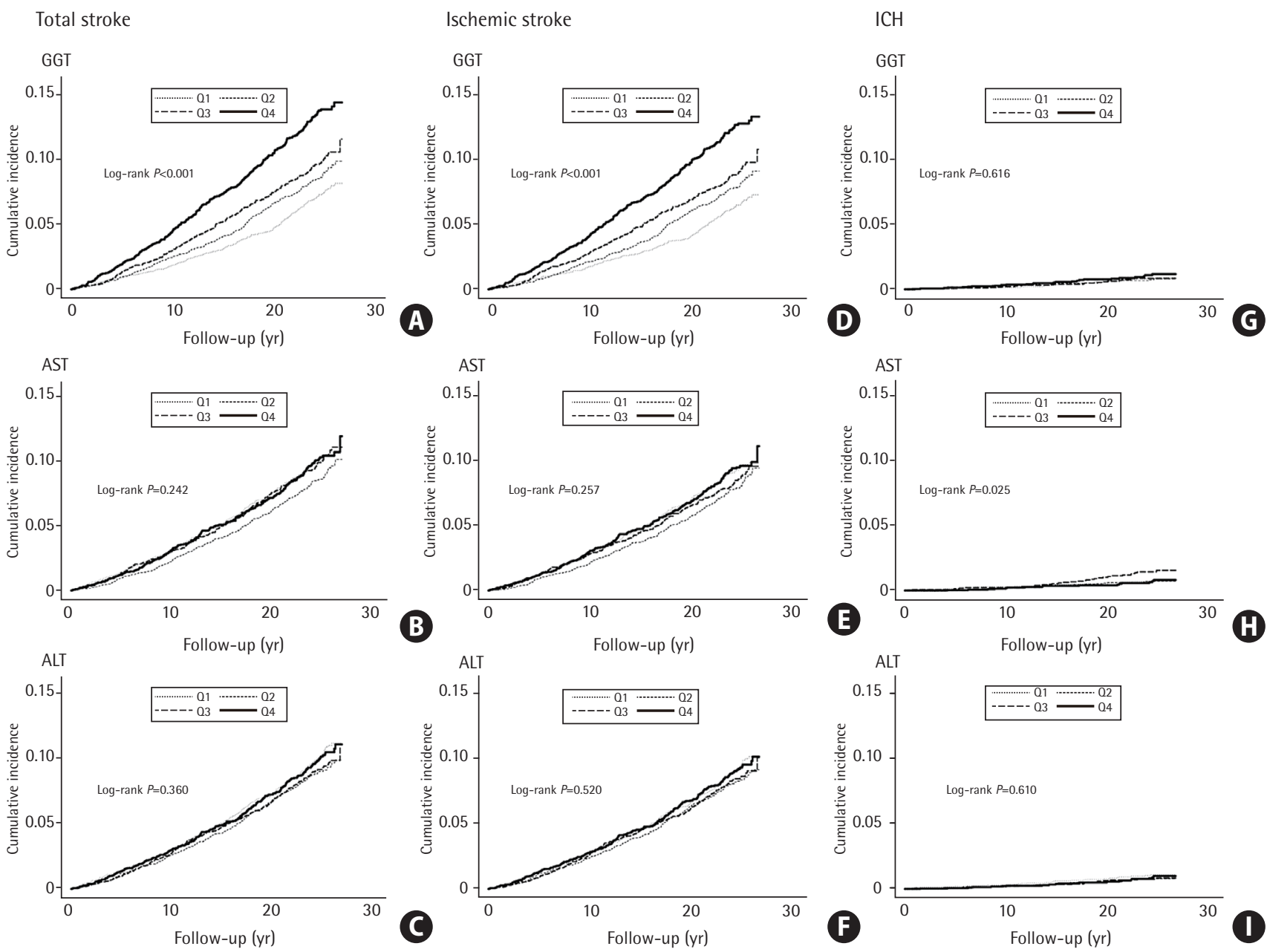

Figure 1. Kaplan-Meier estimates of incidence of total stroke, ischemic stroke and intracerebral hemorrhage (ICH) by quartile of gamma-glutamyl transpeptidase (GGT), aspartate aminotransferase (AST), and alanine aminotransferase (ALT). Kaplan-Meier curve for cumulative incidence of total stroke (A, B, C), ischemic stroke (D, E, F), and ICH (G, H, I) by quartile of GGT, AST, and ALT in Atherosclerosis Risk in Communities (ARIC) Visit 2 (1990-1992) through December 31, 2016. 
death, proportional sub-distribution hazards model by Fine and Grey with a competing risk in the analysis method was applied.

All reported $P$-values are two-sided and $P$-values of $<0.05$ were considered statistically significant. Analyses were conducted using Stata/SE 15.0 software (Stata Corp LP, College Station, TX, USA).

\section{Ethics approval}

The authors did not interact with human subjects per say. This study acquired de-identified participant data from the ARIC study. The ARIC study has been approved by the Institutional Review Boards (IRB) at all participating institutions: University of North Carolina at Chapel Hill IRB, Wake Forest University IRB, Johns Hopkins University IRB, University of Minnesota IRB and University of Mississippi Medical Center IRB. Written informed consent was obtained from all study participants. All methods were carried out in accordance with the relevant guidelines and regulations for human subject research, in accordance with the Declaration of Helsinki (1989).

\section{Results}

\section{Characteristics of the participants}

Our final sample included 12,588 ARIC participants, 56.9 years old on average at baseline (ARIC visit 2, 1990 to 1992), 56.3\% women and 24\% blacks. Table 1 shows the baseline characteristics of the study population by sex-specific quartiles of GGT levels. Characteristics of the study population by AST and ALT levels are shown in Supplementary Tables 1 and 2, the results are similar to those observed by GGT quartiles (Table 1), but the differences across quartiles are less pronounced. Levels of AST and ALT were not significantly different between participants with and without an incident stroke, while median GGT was higher among participants with incident stroke compared to those without stroke: 25.0 (IQR, 17.0 to 38.0) vs. 21.0 (IQR, 14.0 to 33.0), $P<0.001$ (Table not shown).

During a median follow-up of 24.2 years (IQR, 16.7 to 25.4), there were 1,012 incident strokes, among them, 922 (91.1\%) ischemic strokes and 90 (8.9\%) ICH events. The cumulative incidence of total stroke, by quartiles of GGT, AST, and ALT is shown in Figure $1 \mathrm{~A}-\mathrm{C}$, and of ischemic stroke and $\mathrm{ICH}$ in Figure

Table 2. HR (95\% Cl) for incident total stroke by sex-specific quartiles of liver enzymes at baseline, the ARIC study, visit 2, 1990-1992

\begin{tabular}{|c|c|c|c|c|}
\hline Variable & n/total no. & Model 1 & Model 2 & Model 3 \\
\hline \multicolumn{5}{|l|}{ AST } \\
\hline 01 & $307 / 3,731$ & 1 (reference) & 1 (reference) & 1 (reference) \\
\hline 02 & $251 / 3,411$ & $0.83(0.70-0.98)$ & $0.87(0.73-1.03)$ & $0.89(0.76-1.06)$ \\
\hline 03 & $223 / 2,662$ & $0.94(0.79-1.11)$ & $1.00(0.84-1.19)$ & $1.01(0.85-1.20)$ \\
\hline 04 & $231 / 2,784$ & $0.96(0.81-1.14)$ & $1.03(0.86-1.22)$ & $1.00(0.84-1.19)$ \\
\hline$P$ for linear trend & & 0.871 & 0.520 & 0.709 \\
\hline \multicolumn{5}{|l|}{ ALT } \\
\hline 01 & $285 / 3,404$ & 1 (reference) & 1 (reference) & 1 (reference) \\
\hline 02 & $239 / 3,159$ & $0.85(0.72-1.01)$ & $0.87(0.73-1.03)$ & $0.86(0.72-1.02)$ \\
\hline 03 & $243 / 3,108$ & $0.88(0.75-1.05)$ & $0.90(0.76-1.08)$ & $0.86(0.72-1.02)$ \\
\hline 04 & $245 / 2,917$ & $1.05(0.88-1.25)$ & $1.06(0.89-1.26)$ & $0.93(0.78-1.11)$ \\
\hline$P$ for linear trend & & 0.617 & 0.571 & 0.390 \\
\hline \multicolumn{5}{|l|}{ GGT } \\
\hline 01 & $228 / 3,618$ & 1 (reference) & 1 (reference) & 1 (reference) \\
\hline 02 & $214 / 2,890$ & $1.08(0.90-1.30)$ & $1.04(0.86-1.25)$ & $0.95(0.79-1.15)$ \\
\hline 03 & $250 / 3,083$ & $1.19(0.99-1.43)$ & $1.11(0.93-1.34)$ & $0.96(0.80-1.16)$ \\
\hline 04 & $320 / 2,997$ & $1.66(1.40-1.98)$ & $1.53(1.28-1.83)$ & $1.21(1.01-1.46)$ \\
\hline$P$ for linear trend & & $<0.001$ & $<0.001$ & 0.033 \\
\hline
\end{tabular}

The ARIC study population, visit 2, 1990-1992 through December 31, 2016. AST levels (U/L): men $01<12,02$ 12-15, 03 16-21, 04 $\geq 25$; women $01<16$, 02 16-18, 03 19-21, $04 \geq 22$. ALT levels (U/L): men $01<18,02$ 18-21, 03 21-25, $04 \geq 22$; women $01<12,02$ 10-12, 03 13-16, $04 \geq 17$. GGT levels (U/L): men $01<18,02$ 18-24, 03 25-37, 04 >38; women $01<13,02$ 13-17, 03 18-28, $04 \geq 29$. Model 1: Adjusted for age, sex, race-center; Model 2: Model 1+body mass index, smoking status, drinking status, education level; Model 3: Model 2+systolic blood pressure, hypertension medication, prevalent coronary heart disease, diabetes, high-density lipoprotein, total cholesterol, and lipids medication.

HR, hazard ratio; Cl, confidence interval; ARIC, Atherosclerosis Risk in Communities; AST, aspartate aminotransferase; ALT, alanine aminotransferase; GGT, gamma-glutamyl transpeptidase. 
Table 3. HR (95\% Cl) for incident ischemic stroke by sex-specific quartiles of liver enzymes, the ARIC study, visit 2, 1990-1992

\begin{tabular}{|c|c|c|c|c|}
\hline Variable & n/total no. & Model 1 & Model 2 & Model 3 \\
\hline \multicolumn{5}{|l|}{ AST } \\
\hline 01 & $284 / 3,731$ & 1 (reference) & 1 (reference) & 1 (reference) \\
\hline 02 & $231 / 3,411$ & $0.83(0.70-0.99)$ & $0.87(0.73-1.03)$ & $0.90(0.75-1.07)$ \\
\hline 03 & $192 / 2,662$ & $0.87(0.72-1.04)$ & $0.93(0.77-1.12)$ & $0.94(0.78-1.13)$ \\
\hline 04 & $215 / 2,784$ & $0.97(0.81-1.15)$ & $1.03(0.86-1.24)$ & $1.01(0.85-1.21)$ \\
\hline$P$ for linear trend & & 0.725 & 0.670 & 0.860 \\
\hline \multicolumn{5}{|l|}{ ALT } \\
\hline 01 & $256 / 3,404$ & 1 (reference) & 1 (reference) & 1 (reference) \\
\hline 02 & $219 / 3,159$ & $0.87(0.72-1.04)$ & $0.88(0.74-1.06)$ & $0.87(0.73-1.05)$ \\
\hline 03 & $223 / 3,108$ & $0.90(0.75-1.08)$ & $0.92(0.77-1.10)$ & $0.87(0.73-1.05)$ \\
\hline 04 & $224 / 2,917$ & $1.07(0.90-1.28)$ & $1.07(0.89-1.29)$ & $0.94(0.78-1.13)$ \\
\hline$P$ for linear trend & & 0.476 & 0.467 & 0.466 \\
\hline \multicolumn{5}{|l|}{ GGT } \\
\hline 01 & $202 / 3,618$ & 1 (reference) & 1 (reference) & 1 (reference) \\
\hline 02 & $195 / 2,890$ & $1.11(0.91-1.35)$ & $1.06(0.87-1.30)$ & $0.97(0.80-1.19)$ \\
\hline 03 & $230 / 3,083$ & $1.24(1.02-1.50)$ & $1.15(0.95-1.40)$ & $0.99(0.81-1.21)$ \\
\hline 04 & $295 / 2,997$ & $1.74(1.44-2.09)$ & $1.59(1.32-1.92)$ & $1.25(1.03-1.52)$ \\
\hline$P$ for linear trend & & $<0.001$ & $<0.001$ & 0.020 \\
\hline
\end{tabular}

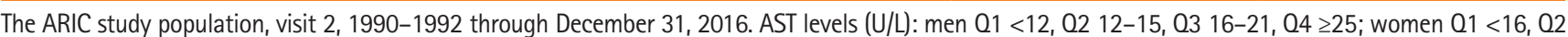
16-18, 03 19-21, 04 222. ALT levels (U/L): men $01<18,02$ 18-21, 03 21-25, $04 \geq 22$; women $01<12,02$ 10-12, 03 13-16, 04 ⒘ GGT levels (U/L): men $01<18,02$ 18-24, 03 25-37, 04 38; women $01<13$, 02 13-17, 03 18-28, 04 229. Model 1: Adjusted for age, sex, race-center; Model 2: Model 1+body mass index, smoking status, drinking status, education level; Model 3: Model 2+systolic blood pressure, hypertension medication, prevalent coronary heart disease (CHD), diabetes, high-density lipoprotein, total cholesterol, and lipids medication. Hypertension medication, diabetes, smoking, drinking, lipids medication, and $\mathrm{CHD}$ were included in Models 2-3 as time-varying variables.

$\mathrm{HR}$, hazard ratio; $\mathrm{Cl}$, confidence interval; ARIC, Atherosclerosis Risk in Communities; AST, aspartate aminotransferase; ALT, alanine aminotransferase; GGT, gamma-glutamyl transpeptidase.

\section{D-F and G-I, respectively.}

Participants in the third and fourth quartiles of GGT had a higher risk of total stroke compared to those in the lowest quartiles (Table 2). The adjusted HRs (95\% confidence intervals [Cls]) for total stroke (Model 3) were $0.98(95 \% \mathrm{Cl}, 0.81$ to 1.19), $1.02(95 \% \mathrm{Cl}, 0.85$ to 1.23$)$, and $1.35(95 \% \mathrm{Cl}, 1.12$ to 1.62) for the 2nd, 3rd, and 4th GGT quartile respectively, compared to those in the 1st quartile ( $P$ for trend $<0.001$ ). Similar findings were observed for ischemic stroke (Table 3).

For AST, compared to individuals in lowest quartiles, those in the third quartile had an increased risk of ICH (adjusted HR, $1.82 ; 95 \% \mathrm{Cl}, 1.06$ to 3.13) (Table 4). No association was found between ALT and incidence of stroke, regardless of the type of stroke (Tables 2-4).

Restricted cubic spline models showed a dose-response, positive, nearly linear association between GGT levels and the risk of total stroke (Figure 2A) and ischemic stroke (Figure 2D), but not for ICH (Figure 2G). However, no significant associations of AST and ALT with total stroke were found (Figure 2B and C). A non-linear association between AST and ICH, but not for isch- emic stroke were observed (Figure $2 \mathrm{H}$ and $\mathrm{E}$, respectively). No association was found for ALT regardless of the type of stroke (Figure $2 \mathrm{C}, \mathrm{F}$, and I). In the sensitivity analysis excluding individuals with NAFLD at baseline, the results were somewhat attenuated but remained significant for GGT (Table 5, Model 3).

In addition, in the proportional sub-distribution hazards analysis for incident stroke with a competing risk of death, significant correlations remained for the fourth quartiles of GGT in total and ischemic stroke patients, and for the third quartiles of AST in ICH patients, compared to the first quartile (Table 6). The adjusted HRs for total stroke (Model 3) were $1.21(95 \% \mathrm{Cl}$, 1.01 to 1.46$)$ and $1.25(95 \% \mathrm{Cl}, 1.03$ to 1.51$)$ for the 4th GGT quartile in total and ischemic stroke respectively, compared to those in the 1st quartile. The adjusted HRs was $1.92(95 \% \mathrm{Cl}$, 1.12 to 3.27 ) for the 3th AST quartile in $\mathrm{ICH}_{\text {, compared to }}$ those in the 1st quartile.

\section{Discussion}

In the ARIC community-based population with nearly 25 years 
Table 4. HR (95\% Cl) for incident ICH by sex-specific quartiles of liver enzymes at baseline, the ARIC study, visit 2, 1990-1992

\begin{tabular}{|c|c|c|c|c|}
\hline Variable & n/total no. & Model 1 & Model 2 & Model 3 \\
\hline \multicolumn{5}{|l|}{ AST } \\
\hline 01 & $23 / 3,731$ & 1 (reference) & 1 (reference) & 1 (reference) \\
\hline 02 & $20 / 3,411$ & $0.89(0.49-1.62)$ & $0.90(0.49-1.65)$ & $0.89(0.49-1.63)$ \\
\hline 03 & $31 / 2,662$ & $1.77(1.03-3.04)$ & $1.87(1.08-3.22)$ & $1.85(1.07-3.18)$ \\
\hline 04 & $16 / 2,784$ & $0.89(0.47-1.68)$ & $0.95(0.50-1.80)$ & $0.91(0.48-1.73)$ \\
\hline$P$ for linear trend & & 0.568 & 0.426 & 0.500 \\
\hline \multicolumn{5}{|l|}{ ALT } \\
\hline 01 & $29 / 3,404$ & 1 (reference) & 1 (reference) & 1 (reference) \\
\hline 02 & $20 / 3,159$ & $0.87(0.72-1.04)$ & $0.88(0.74-1.06)$ & $0.87(0.73-1.05)$ \\
\hline 03 & $20 / 3,108$ & $0.90(0.75-1.08)$ & $0.92(0.77-1.10)$ & $0.87(0.73-1.05)$ \\
\hline 04 & $21 / 2,917$ & $1.07(0.90-1.28)$ & $1.07(0.89-1.29)$ & $0.94(0.78-1.13)$ \\
\hline$P$ for linear trend & & 0.531 & 0.683 & 0.587 \\
\hline \multicolumn{5}{|l|}{ GGT } \\
\hline 01 & $26 / 3,618$ & 1 (reference) & 1 (reference) & 1 (reference) \\
\hline 02 & $19 / 2,890$ & $1.11(0.91-1.35)$ & $1.06(0.87-1.30)$ & $0.97(0.80-1.19)$ \\
\hline 03 & $20 / 3,083$ & $1.24(1.02-1.50)$ & $1.15(0.95-1.40)$ & $0.99(0.81-1.21)$ \\
\hline Q4 & $25 / 2,997$ & $1.74(1.44-2.09)$ & $1.59(1.32-1.92)$ & $1.25(1.03-1.52)$ \\
\hline$P$ for linear trend & & 0.842 & 0.900 & 0.784 \\
\hline
\end{tabular}

ARIC study population, visit 2, 1990-1992 through December 31, 2016. AST levels (U/L): men $01<12,02$ 12-15, 03 16-21, $04 \geq 25$; women $01<16$, 02 16-

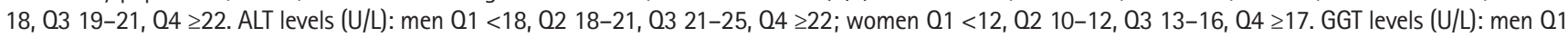
$<18,02$ 18-24, 03 25-37, 04 238; women $01<13,02$ 13-17, 03 18-28, 04 29. Model 1: Adjusted for age, sex, race-center; Model 2: Model 1+body mass index, smoking status, drinking status, education level; Model 3: Model 2+systolic blood pressure, hypertension medication, prevalent coronary heart disease, diabetes, high-density lipoprotein, total cholesterol, and lipids medication.

$\mathrm{HR}$, hazard ratio; $\mathrm{Cl}$, confidence interval; ICH, intracerebral hemorrhage; ARIC, Atherosclerosis Risk in Communities; AST, aspartate aminotransferase; ALT, alanine aminotransferase; GGT, gamma-glutamyl transpeptidase.

of follow-up, higher baseline GGT levels were associated with a significant increase in the risk of stroke, overall and for ischemic stroke, but not for ICH. In addition, the higher AST levels were associated with increased risk of $\mathrm{ICH}$, but not ischemic stroke. The associations remained significant after sub-analysis with death as a competing risk factor.

The association between GGT and risk of stroke remained significant after excluding people with NAFLD. Although the mechanisms by which higher serum GGT levels increase the risk of stroke have not been elucidated, alteration in glutamate concentration might explain the role of GGT in the pathology of cardiovascular diseases, especially in ischemic stroke. ${ }^{17}$ In addition, in the present study, higher levels of AST were associated with increased risk of $\mathrm{ICH}$, but this result is based on a relatively small number of events (90 cases of $\mathrm{ICH}$ ). Future studies in a larger numbers of events are needed in order to verify this association.

The results of studies that focused on the associations between GGT, AST, ALT levels and stroke incidence, have been inconsistent. ${ }^{5,6} \mathrm{~A}$ Korean population-based cohort study including 456,100 participants with up to 11 years of follow-up, GGT was a predictor of ischemic and hemorrhagic stroke, as well as subarachnoid hemorrhage. ${ }^{24}$ The reported association was significant independently from alcohol consumption and other risk factors. In addition, the association was observed more consistently in patients with ischemic stroke than hemorrhagic stroke. Nevertheless, in contrast with the current study, no correlations for AST and ALT were found, and the follow-up period was shorter compared to the ARIC study. To the best of our knowledge, correlations found in our study have not been reported in the USA population, which has different characteristics from the Korean population. Weikert et al. ${ }^{5}$ demonstrated a link between GGT, but not ALT, and stroke in middle-aged German adults, suggesting that these two enzymes have different roles in the development of stroke. In addition, the study showed an increased risk for ICH with higher ALT. In contrast to the current study, AST was not measured in this study. As mentioned by the authors, the inverse relation between ALT and ischemic stroke was not an expected result. However, we didn't find evidence for a similar association in the present study. An important limitation of the study by Weikert et al. ${ }^{5}$ is the limited number of cases (353 strokes) and the relatively 
Total stroke
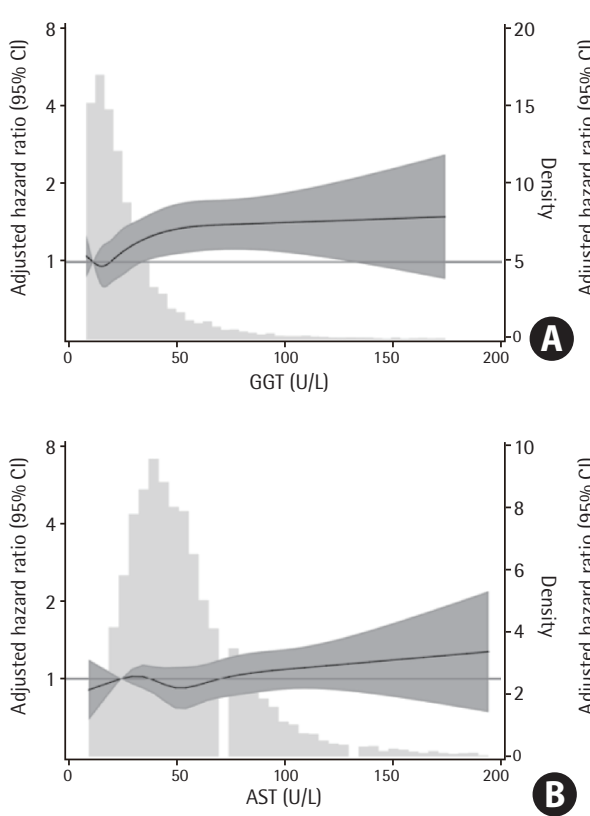

B

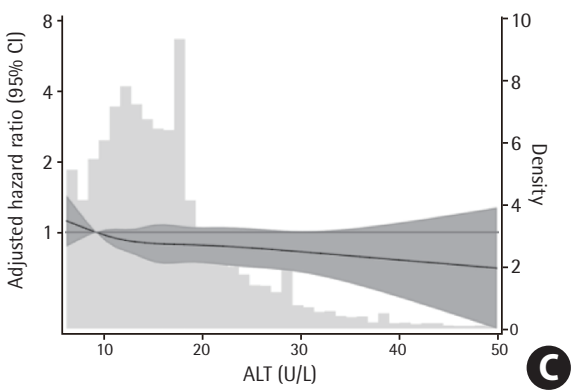

Ischemic stroke

$\mathrm{ICH}$
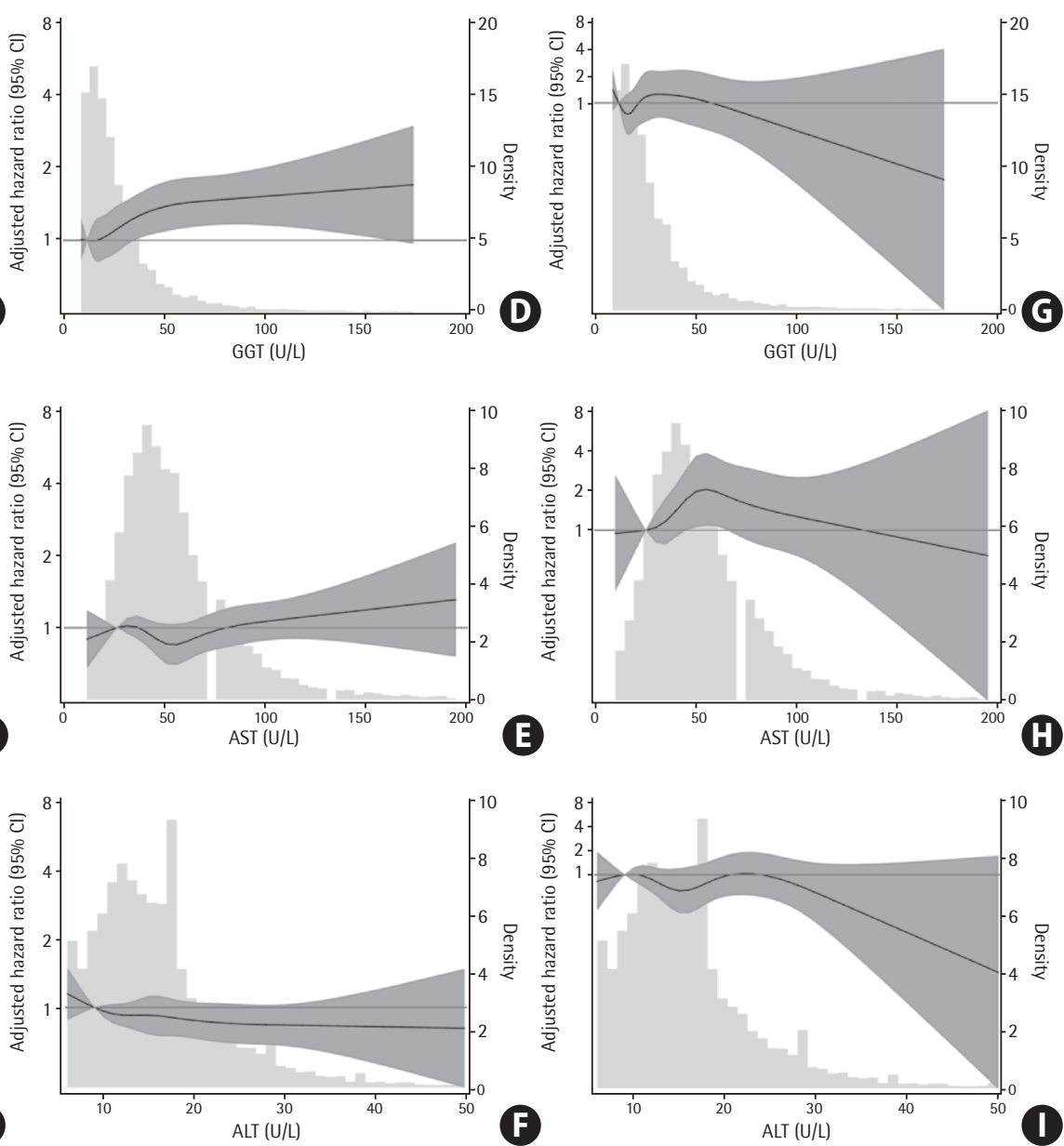

Figure 2. Adjusted hazard ratio (HR; 95\% confidence interval [CI]) for incidence of total stroke, ischemic stroke and intracerebral hemorrhage (ICH) by levels of gamma-glutamyl transpeptidase (GGT), aspartate aminotransferase (AST), and alanine aminotransferase (ALT). Adjusted HR (95\% Cl) for incident total stroke $(A, B, C)$, ischemic stroke (D, E, F), and ICH $(G, H, I)$ by baseline GGT, AST and ALT. Baseline biomarkers were modeled using restricted cubic splines (solid lines) with knots at the 5th, 27.5th, 50th, 72.5th, and 95th percentiles. Gray shade represents the $95 \% \mathrm{Cl}$. Models are centered at the 10 th percentile of each marker and are adjusted for age, sex, race-center, education level, smoking status, body mass index, drinking status, diabetes, high-density lipoprotein, total cholesterol, systolic blood pressure, hypertension medication, lipid-lowing medication, and coronary heart disease.

short follow-up period (median 8.2 years). Similarly to our results regarding AST, Kim et al. ${ }^{6}$ reported no associations between ischemic stroke and both AST and ALT levels, but positive associations between aminotransferase enzymes and ICH in a large 10-year prospective study comprising 108,464 participants of Korean men. These associations were consistent regardless of the level of obesity, blood pressure, fasting glucose, and alcohol intake. However, the serum aminotransferase assay used in the study was not standardized.

In both ischemic stroke and $\mathrm{ICH}_{\text {, glutamate excitotoxicity }}$ has been shown as one of the major contributors to neuronal cell death and bad neurological outcome. ${ }^{2,3,25,26}$ In the present study, three liver enzymes-AST, ALT, and GGT-were chosen for the evaluation of associations between their serum levels and incidence of stroke, based on their role in regulating free blood glutamate levels, and the existing literature regarding the role of glutamate levels in neuroprotection. ${ }^{29,26,27}$ We found no evidence of significant associations between AST and ALT levels and incident ischemic stroke, which accounted for $92 \%$ of all stroke events in ARIC participants. Nevertheless, AST levels were associated with ICH.

Since the neuroprotective effect of AST and ALT has been shown in a few pre-clinical and clinical studies among ischemic stroke patients with increased levels of glutamate, it is possible that AST and ALT levels at the time of the stroke have a neuroprotective effect, but do not significantly influence the 
Table 5. HR (95\% Cl) for incident total stroke by sex-specific quartiles of liver enzymes at baseline after exclusion of individuals with NAFLD at baseline

\begin{tabular}{|c|c|c|c|c|}
\hline Variable & n/total no. & Model 1 & Model 2 & Model 3 \\
\hline \multicolumn{5}{|l|}{ AST } \\
\hline 01 & $307 / 3,730$ & 1 (reference) & 1 (reference) & 1 (reference) \\
\hline 02 & $250 / 3,405$ & $0.83(0.70-0.98)$ & $0.87(0.73-1.03)$ & $0.89(0.76-1.06)$ \\
\hline 03 & $222 / 2,647$ & $0.93(0.79-1.11)$ & $1.00(0.84-1.19)$ & $1.01(0.85-1.20)$ \\
\hline 04 & $182 / 2,106$ & $0.96(0.80-1.16)$ & $1.04(0.86-1.25)$ & $1.03(0.86-1.24)$ \\
\hline Pfor linear trend & & 0.835 & 0.511 & 0.575 \\
\hline \multicolumn{5}{|l|}{ ALT } \\
\hline 01 & $284 / 3,395$ & 1 (reference) & 1 (reference) & 1 (reference) \\
\hline 02 & $237 / 3,139$ & $0.85(0.72-1.01)$ & $0.87(0.73-1.03)$ & $0.86(0.72-1.02)$ \\
\hline 03 & $241 / 3,067$ & $0.89(0.75-1.06)$ & $0.91(0.76-1.08)$ & $0.86(0.72-1.02)$ \\
\hline 04 & $199 / 2,287$ & $1.06(0.89-1.27)$ & $1.07(0.89-1.29)$ & $0.94(0.78-1.13)$ \\
\hline$P$ for linear trend & & 0.658 & 0.615 & 0.399 \\
\hline \multicolumn{5}{|l|}{ GGT } \\
\hline 01 & $227 / 3,574$ & 1 (reference) & 1 (reference) & 1 (reference) \\
\hline 02 & $211 / 2,831$ & $1.08(0.89-1.30)$ & $1.04(0.86-1.25)$ & $0.94(0.78-1.14)$ \\
\hline 03 & $245 / 2,944$ & $1.21(1.01-1.45)$ & $1.13(0.93-1.36)$ & $0.97(0.80-1.17)$ \\
\hline 04 & $278 / 2,539$ & $1.65(1.38-1.98)$ & $1.51(1.25-1.82)$ & $1.19(0.98-1.44)$ \\
\hline Pfor linear trend & & $<0.001$ & $<0.001$ & 0.068 \\
\hline
\end{tabular}

Atherosclerosis Risk in Communities (ARIC) study population, visit 2, 1990-1992 through December 31, 2016. AST levels (U/L): men 01 <12, 02 12-15, 03

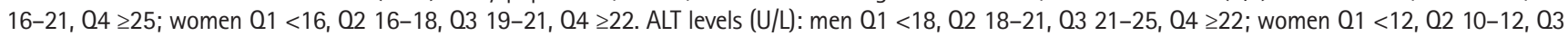
13-16, 04 17. GGT levels (U/L): men $01<18,02$ 18-24, 03 25-37, $04 \geq 38$; women $01<13,02$ 13-17, 03 18-28, $04 \geq 29$. Model 1: Adjusted for age, sex, race-center; Model 2: Model 1+body mass index, smoking status, drinking status, education level; Model 3: Model 2+systolic blood pressure, hypertension medication, prevalent coronary heart disease, diabetes, high-density lipoprotein, total cholesterol, and lipids medication. Six hundred ninety-seven ARIC participants with NAFLD defined as elevated AST or ALT, and, consuming 21 standard drinks on average per week in men or 14 standard drinks on average per week in women.

$\mathrm{HR}$, hazard ratio; $\mathrm{Cl}$, confidence interval; NAFLD, non-alcoholic fatty liver disease; AST, aspartate aminotransferase; ALT, alanine aminotransferase; GGT, gamma-glutamyl transpeptidase.

risk for development of ischemic stroke. In addition, previous publications have reported stronger associations of AST versus ALT levels with I outcome after stroke, and significantly stronger anti-glutamatergic activity of AST compared with ALT. These results suggest that AST and ALT might have different roles in association with the risk of ischemic stroke and $\mathrm{ICH}^{9,11}$ Additional studies are needed to further test the role of blood glutamate, AST and ALT levels as risk factors for incident stroke, in order to clarify the association between glutamate regulation and occurrence of stroke. In particular, studies using systems biology are needed to better understand the interplay between glutamate levels and liver enzymes activity in healthy population, as opposed to their association in the presence of acute stroke.

This study has several notable strengths, including the use of a large community-based biracial population, extensive assessment of cardiovascular risk factors including time-varying data on risk factors, physician-adjudicated events as ischemic stroke or ICH, and approximately 25 years of follow-up. Nonetheless, there are some limitations. Blood glutamate levels were not available; therefore, we could not study the direct association between glutamate and its metabolites and risk of stroke. Also, data on liver enzymes was available for all participants at baseline (1990 to 1992) but not at the exact time of stroke incidence and only one measure of liver enzymes was available. Given the large intra-individual variation in AST, ALT, and GGT, the use of a single measure of these enzymes may bias the results towards the null. ${ }^{28,29}$ Smoking status and alcohol use were self-reported so the reporting bias cannot be excluded. Last, the number of ICH cases was relatively small and therefore we had limited power for ICH as outcome.

\section{Conclusions}

In conclusion, elevated levels of GGT (within normal levels), independent of liver disease, are associated with higher risk of incident stroke overall and ischemic stroke, but not ICH. Future studies including measurements of enzyme activity and plasma 
Table 6. SHR (95\% CI) for incident stroke with a competing risk of death by sex-specific quartiles of liver enzymes at baseline, the ARIC study, visit 2, 1990-1992

\begin{tabular}{|c|c|c|c|}
\hline \multirow{2}{*}{ Variable } & \multicolumn{3}{|c|}{ Model 3} \\
\hline & Total stroke & Ischemic stroke & $\mathrm{ICH}$ \\
\hline \multicolumn{4}{|l|}{ AST } \\
\hline 01 & 1 (reference) & 1 (reference) & 1 (reference) \\
\hline \multirow[t]{2}{*}{02} & $0.94(0.79-1.11)$ & $0.94(0.79-1.12)$ & $0.94(0.52-1.71)$ \\
\hline & $P=0.453$ & $P=0.512$ & $P=0.846$ \\
\hline \multirow[t]{2}{*}{03} & $1.04(0.88-1.24)$ & $0.97(0.81-1.17)$ & $1.92(1.12-3.27)$ \\
\hline & $P=0.624$ & $P=0.789$ & $P=0.017$ \\
\hline \multirow[t]{2}{*}{ Q4 } & $1.00(0.84-1.19)$ & $1.02(0.85-1.22)$ & $0.90(0.47-1.71)$ \\
\hline & $P=0.977$ & $P=0.860$ & $P=0.751$ \\
\hline \multicolumn{4}{|l|}{ ALT } \\
\hline 01 & 1 (reference) & 1 (reference) & 1 (reference) \\
\hline \multirow[t]{2}{*}{02} & $0.91(0.76-1.08)$ & $0.93(0.77-1.11)$ & $0.76(0.43-1.36)$ \\
\hline & $P=0.278$ & $P=0.428$ & $P=0.359$ \\
\hline \multirow[t]{2}{*}{ Q3 } & $0.90(0.76-1.08)$ & $0.92(0.76-1.11)$ & $0.79(0.45-1.39)$ \\
\hline & $P=0.253$ & $P=0.375$ & $P=0.412$ \\
\hline \multirow[t]{2}{*}{04} & $0.94(0.78-1.12)$ & $0.95(0.79-1.15)$ & $0.86(0.48-1.54)$ \\
\hline & $P=0.474$ & $P=0.601$ & $P=0.608$ \\
\hline \multicolumn{4}{|l|}{ GGT } \\
\hline 01 & 1 (reference) & 1 (reference) & 1 (reference) \\
\hline \multirow[t]{2}{*}{$\mathrm{Q} 2$} & $1.00(0.83-1.20)$ & $1.01(0.83-1.24)$ & $0.87(0.47-1.58)$ \\
\hline & $P=0.971$ & $P=0.889$ & $P=0.636$ \\
\hline \multirow[t]{2}{*}{ Q3 } & 0.99 (0.82-1.19) & $1.01(0.83-1.23)$ & $0.80(0.44-1.48)$ \\
\hline & $P=0.901$ & $P=0.895$ & $P=0.483$ \\
\hline \multirow[t]{2}{*}{04} & $1.21(1.01-1.46)$ & $1.25(1.03-1.51)$ & $0.97(0.54-1.72)$ \\
\hline & $P=0.038$ & $P=0.026$ & $P=0.907$ \\
\hline
\end{tabular}

ARIC study population, visit 2, 1990-1992 through December 31, 2016. AST levels (U/L): men $01<12,02$ 12-15, 03 16-21, $04 \geq 25$; women $01<16$, 02 16-

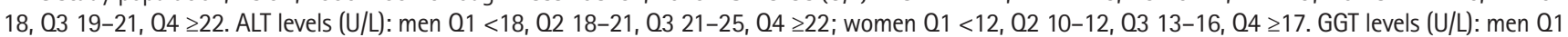
$<18,02$ 18-24, 03 25-37, $04 \geq 38$; women $01<13,02$ 13-17, 03 18-28, $04 \geq 29$. Model 3: Model 2+systolic blood pressure, hypertension medication, prevalent coronary heart disease, diabetes, high-density lipoprotein, total cholesterol, and lipids medication.

SHR, sub-hazard ratio; $\mathrm{Cl}$, confidence interval; ARCl, Atherosclerosis Risk in Communities; ICH, intracerebral hemorrhage; AST, aspartate aminotransferase; ALT, alanine aminotransferase; GGT, gamma-glutamyl transpeptidase.

levels of glutamate, are required to further evaluate to which extent the observed association could be related to liver enzymes ability to increase glutamate levels.

\section{Supplementary materials}

Supplementary materials related to this article can be found online at https://doi.org/10.5853/10.5853/jos.2020.00290.

\section{Disclosure}

The authors thank the staff and participants of the ARIC study for their important contributions. This work was supported by
NIH/NIDDK grant R01DK089174 to Dr. Elizabeth Selvin. Reagents for the ALT, AST, and GGT assays were donated by Roche Diagnostics.

\section{Acknowledgments}

The Atherosclerosis Risk in Communities study has been funded in whole or in part with Federal funds from the National Heart, Lung, and Blood Institute, National Institutes of Health, Department of Health and Human Services, under Contract nos. (HHSN268201700001I, HHSN268201700003I, HHSN $268201700005 \mathrm{I}, \mathrm{HHSN} 268201700004 \mathrm{I}, \mathrm{HH}-$ SN2682017000021). Neurocognitive data is collected by U01 
2U01HL096812, 2U01HL096814, 2U01HL096899, 2U01HL096902, 2U01HL096917 from the NIH (NHLBI, NINDS, NIA and NIDCD), and with previous brain MRI examinations funded by R01-HL70825 from the NHLBI. Dr. Selvin was supported by NIH/NIDDK K24DK106414 and R01DK089174 grants. Dr. Lazo was supported by NIH/NIDDK grant R01DK089174.

\section{References}

1. Benjamin EJ, Muntner P, Alonso A, Bittencourt MS, Callaway CW, Carson AP, et al. Heart disease and stroke statistics-2019 update: a report from the American Heart Association. Circulation 2019;139:e56-e528.

2. Jung CS, Lange $B$, Zimmermann $M$, Seifert V. CSF and serum biomarkers focusing on cerebral vasospasm and ischemia after subarachnoid hemorrhage. Stroke Res Treat 2013;2013:560305.

3. Lai TW, Zhang S, Wang YT. Excitotoxicity and stroke: identifying novel targets for neuroprotection. Prog Neurobiol 2014; 115:157-188.

4. Zheng $Y, H u$ FB, Ruiz-Canela M, Clish CB, Dennis C, SalasSalvado J, et al. Metabolites of glutamate metabolism are associated with incident cardiovascular events in the PREDIMED PREvención con Dleta MEDiterránea (PREDIMED) Trial. J Am Heart Assoc 2016;5:e003755.

5. Weikert C, Drogan D, di Giuseppe R, Fritsche A, Buijsse B, Nöthlings $U$, et al. Liver enzymes and stroke risk in middleaged German adults. Atherosclerosis 2013;228:508-514.

6. Kim HC, Kang DR, Nam CM, Hur NW, Shim JS, Jee SH, et al. Elevated serum aminotransferase level as a predictor of intracerebral hemorrhage: Korea Medical Insurance Corporation study. Stroke 2005;36:1642-1647.

7. Eckel RH, Grundy SM, Zimmet PZ. The metabolic syndrome. Lancet 2005;365:1415-1428.

8. Pratt DS, Kaplan MM. Evaluation of abnormal liver-enzyme results in asymptomatic patients. N Engl J Med 2000;342: 1266-1271.

9. Campos F, Rodríguez-Yáñez M, Castellanos M, Arias S, PérezMato $M$, Sobrino $T$, et al. Blood levels of glutamate oxaloacetate transaminase are more strongly associated with good outcome in acute ischaemic stroke than glutamate pyruvate transaminase levels. Clin Sci (Lond) 2011;121:11-17.

10. Khanna S, Briggs Z, Rink C. Inducible glutamate oxaloacetate transaminase as a therapeutic target against ischemic stroke. Antioxid Redox Signal 2015;22:175-186.

11. Boyko M, Stepensky D, Gruenbaum BF, Gruenbaum SE, Melamed I, Ohayon S, et al. Pharmacokinetics of glutamateoxaloacetate transaminase and glutamate-pyruvate transaminase and their blood glutamate-lowering activity in na- ïve rats. Neurochem Res 2012;37:2198-2205.

12. Ndrepepa G, Kastrati A. Gamma-glutamyl transferase and cardiovascular disease. Ann Transl Med 2016;4:481.

13. Tate SS, Meister A. Gamma-glutamyl transpeptidase: cataIytic, structural and functional aspects. Mol Cell Biochem 1981;39:357-368.

14. Hanigan $\mathrm{MH}$. Gamma-glutamyl transpeptidase: redox regulation and drug resistance. Adv Cancer Res 2014;122:103141.

15. Loomba R, Doycheva I, Bettencourt R, Cohen B, Wassel CL, Brenner $D$, et al. Serum $\gamma$-glutamyltranspeptidase predicts all-cause, cardiovascular and liver mortality in older adults. $J$ Clin Exp Hepatol 2013;3:4-11.

16. Kunutsor SK, Apekey TA, Khan H. Liver enzymes and risk of cardiovascular disease in the general population: a metaanalysis of prospective cohort studies. Atherosclerosis 2014; 236:7-17.

17. Koga M, Serritella AV, Messmer MM, Hayashi-Takagi A, Hester LD, Snyder SH, et al. Glutathione is a physiologic reservoir of neuronal glutamate. Biochem Biophys Res Commun 2011; 409:596-602.

18. van der Stel $A X$, van Mourik $A$, Łaniewski $P$, van Putten JP, Jagusztyn-Krynicka EK, Wösten MM. The Campylobacter jejuni RacRS two-component system activates the glutamate synthesis by directly upregulating $\mathrm{Y}$-glutamyltranspeptidase (GGT). Front Microbiol 2015;6:567.

19. Jousilahti $P$, Rastenyte $D$, Tuomilehto J. Serum gamma-glutamyl transferase, self-reported alcohol drinking, and the risk of stroke. Stroke 2000;31:1851-1855.

20. Shimizu Y, Imano H, Ohira T, Kitamura A, Kiyama M, Okada T, et al. Gamma-glutamyltranspeptidase and incident stroke among Japanese men and women: the Circulatory Risk in Communities Study (CIRCS). Stroke 2010;41:385-388.

21. The ARIC investigators. The Atherosclerosis Risk in Communities (ARIC) Study: design and objectives. Am J Epidemiol 1989;129:687-702.

22. Rosamond WD, Folsom AR, Chambless LE, Wang CH, McGovern PG, Howard G, et al. Stroke incidence and survival among middle-aged adults: 9-year follow-up of the Atherosclerosis Risk in Communities (ARIC) cohort. Stroke 1999;30:736-743.

23. Harrell FE. Regression Modeling Strategies: With Applications to Linear Models, Logistic Regression, and Survival Analysis. New York, NY: Springer, 2001.

24. Yang W, Kim CK, Kim DY, Jeong HG, Lee SH. Gamma-glutamyl transferase predicts future stroke: a Korean nationwide study. Ann Neurol 2018;83:375-386.

25. Chandra A, Stone CR, Du X, Li WA, Huber M, Bremer R, et al. The cerebral circulation and cerebrovascular disease III: 
stroke. Brain Circ 2017;3:66-77.

26. Campos $F$, Sobrino $T$, Ramos-Cabrer $P$, Castellanos $M, B$ Banco $M$, Rodríguez-Yáñez $M$, et al. High blood glutamate oxaloacetate transaminase levels are associated with good functional outcome in acute ischemic stroke. J Cereb Blood Flow Metab 2011;31:1387-1393.

27. da Silva-Candal A, Pérez-Díaz A, Santamaría M, Correa-Paz $C$, Rodríguez-Yáñez $M$, Ardá $A$, et al. Clinical validation of blood/brain glutamate grabbing in acute ischemic stroke.
Ann Neurol 2018;84:260-273.

28. Dufour DR, Lott JA, Nolte FS, Gretch DR, Koff RS, Seeff LB. Diagnosis and monitoring of hepatic injury. I. Performance characteristics of laboratory tests. Clin Chem 2000;46:20272049.

29. Lazo M, Selvin E, Clark JM. Brief communication: clinical implications of short-term variability in liver function test results. Ann Intern Med 2008;148:348-352. 
Supplementary Table 1. Characteristics of the study population by quartiles of AST level at baseline, the ARIC study, visit 2, 1990-1992

\begin{tabular}{|c|c|c|c|c|c|c|}
\hline Characteristic & Total & AST (01) & AST (02) & AST (03) & AST (04) & $P$ \\
\hline Number & 12,588 & 3,731 (29.6) & $3,411(27.1)$ & $2,662(21.1)$ & $2,784(22.1)$ & \\
\hline \multicolumn{7}{|l|}{ AST range (U/L) } \\
\hline Male & & $<19$ & $19-21$ & $22-25$ & $\geq 26$ & \\
\hline Female & & $<17$ & $17-19$ & $20-22$ & $\geq 23$ & \\
\hline Age (yr) & $56.9 \pm 5.7$ & $56.5 \pm 5.7$ & $56.9 \pm 5.9$ & $57.2 \pm 5.7$ & $56.9 \pm 5.5$ & $<0.001$ \\
\hline Black (\%) & 24 & 24.2 & 23.5 & 23.2 & 25.2 & 0.287 \\
\hline Female (\%) & 56.3 & 54.6 & 59.6 & 53.7 & 56.9 & $<0.001$ \\
\hline BMI $\left(\mathrm{kg} / \mathrm{m}^{2}\right)$ & $28.0 \pm 5.4$ & $28.0 \pm 5.5$ & $27.7 \pm 5.3$ & $27.8 \pm 5.2$ & $28.6 \pm 5.6$ & \\
\hline Obesity (\%) & 29.1 & 28.3 & 26.7 & 28.2 & 34 & $<0.001$ \\
\hline SBP $(m m ~ H g)$ & $121.2 \pm 18.5$ & $120.2 \pm 18.7$ & $120.6 \pm 18.0$ & $121.6 \pm 18.2$ & $123.0 \pm 19.0$ & $<0.001$ \\
\hline $\mathrm{DBP}(\mathrm{mm} \mathrm{Hg})$ & $72.1 \pm 10.2$ & $71.1 \pm 10.2$ & $71.6 \pm 10.1$ & $72.6 \pm 10.1$ & $73.5 \pm 10.3$ & $<0.001$ \\
\hline Hypertension (\%) & 39.4 & 38 & 37.4 & 38.1 & 44.9 & $<0.001$ \\
\hline Antihypertensive med use (\%) & 32 & 31.3 & 29.9 & 31.3 & 36 & $<0.001$ \\
\hline $\mathrm{HDL}-\mathrm{C}(\mathrm{mg} / \mathrm{dL})$ & $47.0(37.0-59.0)$ & $46.0(37.0-57.0)$ & $48.0(39.0-61.0)$ & $47.0(37.0-59.0)$ & $47.0(37.0-59.5)$ & $<0.001$ \\
\hline LDL-C (mg/dL) & $131.0(108.8-155.4)$ & $130.0(108.2-155.0)$ & $131.0(109.6-154.2)$ & $132.2(109.6-157.2)$ & 130.6 (108.0-155.6) & 0.292 \\
\hline Triglycerides (mg/dL) & $114.0(82.0-162.0)$ & 113.0 (82.0-159.0) & 110.0 (81.0-156.0) & $114.0(81.0-164.0)$ & $119.0(82.0-175.0)$ & $<0.001$ \\
\hline Total cholesterol (mg/dL) & $207.0(183.0-233.0)$ & $204.0(181.0-230.0)$ & $207.0(184.0-232.0)$ & $208.0(184.0-234.0)$ & $209.0(185.0-236.0)$ & $<0.001$ \\
\hline Lipid lowering med use (\%) & 6.3 & 4.9 & 6 & 6.6 & 8.2 & $<0.001$ \\
\hline $\begin{array}{l}\text { Diabetes (self-report, } \\
\text { meds, FG) (\%) }\end{array}$ & 15.6 & 18.1 & 13.1 & 13.6 & 17.5 & $<0.001$ \\
\hline Prevalent CHD (\%) & 5.4 & 6 & 5 & 5.1 & 5.6 & 0.273 \\
\hline Smoking status (\%) & & & & & & $<0.001$ \\
\hline Current & 21.9 & 28.9 & 22.1 & 18.2 & 15.6 & \\
\hline Former & 37.8 & 34.1 & 37.6 & 39.6 & 41.3 & \\
\hline Never & 40.3 & 37 & 40.3 & 42.1 & 43 & \\
\hline Drinking status (\%) & & & & & & $<0.001$ \\
\hline Current & 21.9 & 28.9 & 22.1 & 18.2 & 15.6 & \\
\hline Former & 37.8 & 34.1 & 37.6 & 39.6 & 41.3 & \\
\hline Never & 40.3 & 370. & 40.3 & 42.1 & 43.0 & \\
\hline
\end{tabular}

Values are presented as number (\%), mean \pm standard deviation, or median (interquartile range).

AST, aspartate aminotransferase; ARIC, Atherosclerosis Risk in Communities; BMI, body mass index; SBP, systolic blood pressure; DBP, diastolic blood pressure; HDL-C, high-density lipoprotein cholesterol; LDL-C, low density lipoprotein cholesterol; FG, fasting glucose; CHD, coronary heart disease. 
Supplementary Table 2. Characteristics of the study population by quartiles of ALT level at baseline, the ARIC study, visit 2, 1990-1992

\begin{tabular}{|c|c|c|c|c|c|c|}
\hline Characteristic & Total & ALT (01) & ALT (02) & ALT (03) & ALT (04) & $P$ \\
\hline Number (\%) & 12,588 & $3,404(27.0)$ & $3,159(25.1)$ & $3,108(24.7)$ & 2,917 (23.2) & \\
\hline \multicolumn{7}{|l|}{ ALT range (U/L) } \\
\hline Male & & $<13$ & $13-16$ & $17-22$ & $\geq 23$ & \\
\hline Female & & $<11$ & $11-13$ & $14-17$ & $\geq 18$ & \\
\hline Age (yr) & $56.9 \pm 5.7$ & $57.1 \pm 5.9$ & $57.1 \pm 5.7$ & $56.9 \pm 5.7$ & $56.3 \pm 5.5$ & $<0.001$ \\
\hline Black (\%) & 24 & 26 & 25.1 & 23.3 & 21.3 & $<0.001$ \\
\hline Female (\%) & 56.3 & 59.4 & 56.8 & 51.8 & 56.8 & $<0.001$ \\
\hline BMI $\left(\mathrm{kg} / \mathrm{m}^{2}\right)$ & $28.0 \pm 5.4$ & $26.7 \pm 5.3$ & $27.5 \pm 5.2$ & $28.4 \pm 5.2$ & $29.7 \pm 5.4$ & $<0.001$ \\
\hline Obesity (\%) & 29.1 & 20.1 & 25.5 & 31.4 & 41.2 & $<0.001$ \\
\hline Estrogen (\%) & 25.9 & 27.7 & 27.6 & 23.7 & 24.1 & $<0.001$ \\
\hline Aspirin (\%) & 50.6 & 49.1 & 49.5 & 51.5 & 52.7 & 0.012 \\
\hline $\mathrm{SBP}(\mathrm{mm} \mathrm{Hg})$ & $121.2 \pm 18.5$ & $120.1 \pm 19.5$ & $120.2 \pm 18.5$ & $121.2 \pm 17.8$ & $123.5 \pm 18.0$ & $<0.001$ \\
\hline DBP $(\mathrm{mm} \mathrm{Hg})$ & $72.1 \pm 10.2$ & $70.9 \pm 10.5$ & $71.4 \pm 10.1$ & $72.4 \pm 9.9$ & $73.9 \pm 10.1$ & $<0.001$ \\
\hline Hypertension (\%) & 39.4 & 36.8 & 37.8 & 39.3 & 44.1 & $<0.001$ \\
\hline Antihypertensive med use (\%) & 32 & 29.6 & 30.7 & 32.2 & 35.7 & $<0.001$ \\
\hline $\mathrm{HDL}-\mathrm{C}(\mathrm{mg} / \mathrm{dL})$ & $47.0(37.0-59.0)$ & $50.0(40.0-62.0)$ & $48.0(39.0-61.0)$ & $45.0(37.0-57.0)$ & $44.0(35.0-55.0)$ & $<0.001$ \\
\hline $\mathrm{LDL}-\mathrm{C}(\mathrm{mg} / \mathrm{dL})$ & $131.0(108.8-155.4)$ & $127.6(105.8-152.2)$ & $131.0(108.8-155.6)$ & $132.0(110.0-156.6)$ & $133.5(111.0-157.4)$ & $<0.001$ \\
\hline Triglycerides (mg/dL), median & $114.0(82.0-162.0)$ & $103.0(76.0-144.0)$ & $109.0(79.0-152.0)$ & 115.0 (83.0-167.0) & $133.0(94.5-192.0)$ & $<0.001$ \\
\hline Total cholesterol (mg/dL), median & $207.0(183.0-233.0)$ & $203.0(180.0-228.0)$ & $207.0(184.0-231.0)$ & $208.0(184.0-233.0)$ & $211.0(187.0-238.0)$ & $<0.001$ \\
\hline Lipid lowering med use, \% & 6.3 & 4.5 & 6.2 & 7.5 & 7.1 & $<0.001$ \\
\hline Diabetes (self-report, meds, FG), \% & 15.6 & 11.1 & 12.7 & 16.2 & 23.5 & $<0.001$ \\
\hline Prevalent CHD, \% & 5.4 & 5.8 & 5.7 & 5.4 & 4.7 & 0.184 \\
\hline Smoking status, \% & & & & & & $<0.001$ \\
\hline Current & 21.9 & 28.9 & 22.7 & 19.5 & 15.2 & \\
\hline Former & 37.8 & 33.7 & 37.5 & 38.4 & 42.3 & \\
\hline Never & 40.3 & 37.3 & 39.8 & 42.1 & 42.4 & \\
\hline Drinking status, $\%$ & & & & & & 0.176 \\
\hline Current & 57 & 55.9 & 56.2 & 57.9 & 57.9 & \\
\hline Former & 20.6 & 21.7 & 21.5 & 19.4 & 19.7 & \\
\hline Never & 22.4 & 22.4 & 22.3 & 22.7 & 22.4 & \\
\hline AST (U/L) & $20.0(17.0-23.0)$ & $17.0(15.0-19.0)$ & $19.0(16.0-21.0)$ & $21.0(18.0-23.0)$ & $25.0(21.0-30.0)$ & $<0.001$ \\
\hline ALT (U/L) & $14.0(11.0-19.0)$ & $9.0(7.0-10.0)$ & $13.0(12.0-14.0)$ & $17.0(15.0-19.0)$ & $25.0(21.0-31.0)$ & $<0.001$ \\
\hline GGT (U/L) & $21.0(14.0-33.0)$ & $16.0(12.0-23.0)$ & $19.0(13.0-27.0)$ & $23.0(16.0-34.0)$ & $33.0(22.0-54.0)$ & $<0.001$ \\
\hline
\end{tabular}

Values are presented as number (\%), mean \pm standard deviation, or median (interquartile range). The ARIC study population, visit 2, 1990-1992; end of followup December 31, 2016.

ALT, alanine aminotransferase; ARIC, Atherosclerosis Risk in Communities; BMI, body mass index; SBP, systolic blood pressure; DBP, diastolic blood pressure; HDL-C, high-density lipoprotein cholesterol; LDL-C, low density lipoprotein cholesterol; FG, fasting glucose; CHD, coronary heart disease; AST, aspartate aminotransferase; GGT, gamma-glutamyl transpeptidase. 